\title{
ROBOTIZED CELL OF PREFABRICATED PANELS
}

\author{
J. Penadés, F. Moliner and J. Florez
}

\section{DRAGADOS CONSTRUCCION Av. de Tenerife 4-८, S. Sebastián de los Reyes, 28700 Madrid, Spain.}

\begin{abstract}
A robotized manufacturing cell of pre-fab icated GRC (Glass Reinforced Cement) panels for construction industry has been deve oped by construction company Dragados, S.A. The main contribution of the developed s.'stem is the automatic programming and control of the whole plant. As input serves the architect's 3D-drawing of the building facade done on a CAD system. From the CAD de: ign, the optimum facade to pan-

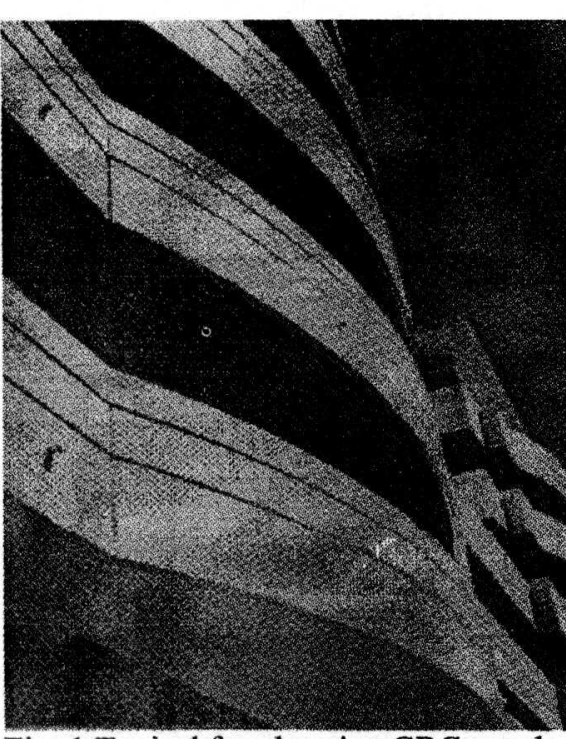

Fig. 1 Typical facade using GRC panels els partition is c btained. In order to manufacture each panel, au omatic task and path planning are performed : or the equipment present in the manufacturing cell: spraying robot, PLCs, control comput :r, etc.

Construction processes done off-site are more suitable to be obotized, since the work takes place in a stru tured environment. A common off-site proces: is the manufacturing of prefabricated parels (Fig.1) which are later assembled on-site. In last years one important material used in this kind of industry has been the Glass Reinf orced Cement (GRC). The GRC material is base $\mathrm{d}$ on mixing cement with small cut glass fiber strips, achieving enough flextraction strength while maintaining light weight. This allows to manufacture large panels $(6 \mathrm{x}$ $3 \mathrm{~m}$ ) of any $3 \mathrm{D}$ geometry with the advantage of easy transportation and easy assembly on site.
\end{abstract}

Keywords: Robots, path planning, prefabrication, automati : control.

\section{PROBLEM STATEMENT}

The manual manufacturing is done using a conventional concentric spraying gun equipped with glass fiber cutting razors. The mortar and the glass fiber strips are projected on a panel mould in two different but simultaneous shots which are mixed in the air and form a spraying cone (Fig. 2). The required final thickness $(1-1.5 \mathrm{~cm})$ of the panel is obtained by progressive spraying into the mould of several layers. Commonly one operator does the spraying process while at the same time 2 or 3 others are compacting. Cycle time for manual manufacturing varies with panel type and size from 15 to $30 \mathrm{~min}$, without taking into account a set of auxiliary operations done before and after projection and compacting.

One of the main requirements is to achieve great uniformity during the spraying process. But in manual production this feature depends on the worker's ability and experience. Another important factors are the working conditions and the environment impact. Workers are faced with a very dirty environment,

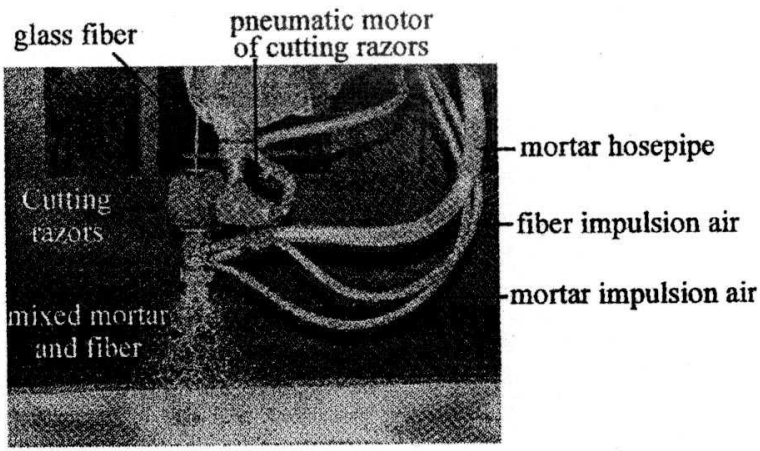

Fig. 2 Concentric spraying gun

which afficts their performance and presents a high risk to the $r$ health too.

Another aspect is the fact that the 3D geometry of panels ch inges very frequently depending on the architect's design. These variations and the building they are rlestined to. Even if small differences between panels are not taken into account series do not exceed 50 units and only in very rare cases amount to a hundred units. 
There are different panels types depending on the type and number of layers to be sprayed. The first layer, which forms the external surface of the resulting panel, is common to all of them. It is done with mortar without fiber. Depending on the remaining layers, there are five distinct types of panels (Fig. 3): a) Plain shell: two more layers of mortar and fiber, b) Shell with ribs: same as plain shell but with stiffening ribs, c) Stud frame: same as plain shell but with a steel frame, d) Shell with insulation: same as plain shell but with insulation sheets, and e) Sandwich: same as plain shell with insulation with and additional GRC top layer.

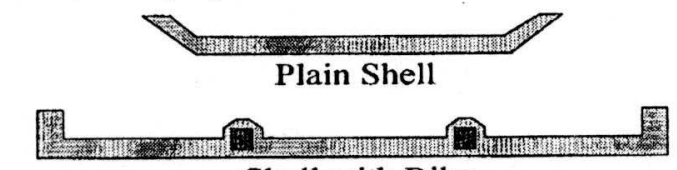

Shell with Ribs
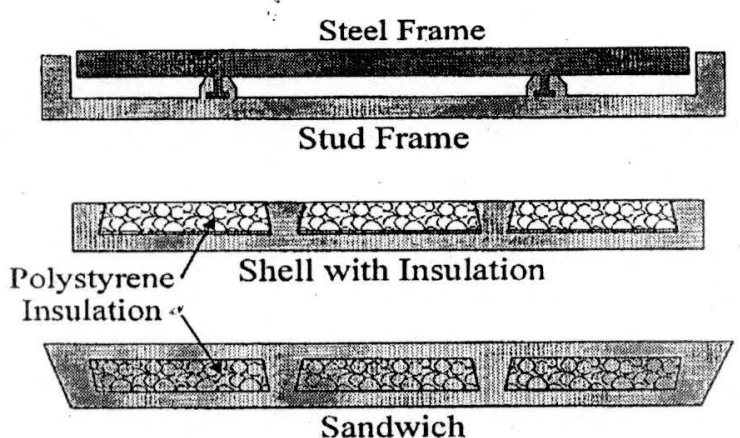

Fig. 3. Different types of GRC panels

\section{OBJECTIVE}

From the preceding section it is clear that some kind of automation which improves flexibility and quality is desirable. Therefore the objective of the automation project has been the design of a robotized system in order to substitute the manual process while improving labor conditions, reducing wasted material, increasing product quality and uniformity, and reducing labor requirements (Fig. 4). The production of GRC panels is done through several stages, of which spraying and compacting are the critical ones. Therefore, automation has focused its attention on this two [1].

Based on the experience obtained through the years of manual production the system is designed flexible enough to cope with small batch size production of different panels, integrating CAD and CAM. A great effort has be made to develop an integrated flexible low-cost system to be used on a range of similar applications, like gluing, sealing, cleaning, etc. Today a highly flexible production unit, which is capable of manufacturing a big variety of small series under quasi-real time request, is crucial for most companies. This can be achieved in the manufacturing environment with the use of Flexible Manufacturing Systems (FMS) under Computer Integrated
Manufacturing (CIM) [2]. This concept has been recently adapted to the construction industry introducing the Computer Integrated Construction (CIC) [3]. The development of an FMS for CIC has to keep in mind the inherent barriers common to these kind of systems: 1) low level of reuse of software and/or hardware, 2) medium level robustness of the developed algorithms under new manufacturing conditions, and specially 3 ) the difficulties of the knowhow transfer between the developing institution and the recipient. This is what has been done in the development of the GRC spraying/compacting cell. The intention right from the beginning was to develop a FMS for a family of different applications related with 3D surface treatment: spraying, painting, cleaning, sealing, etc., being spraying/compacting a particular case.

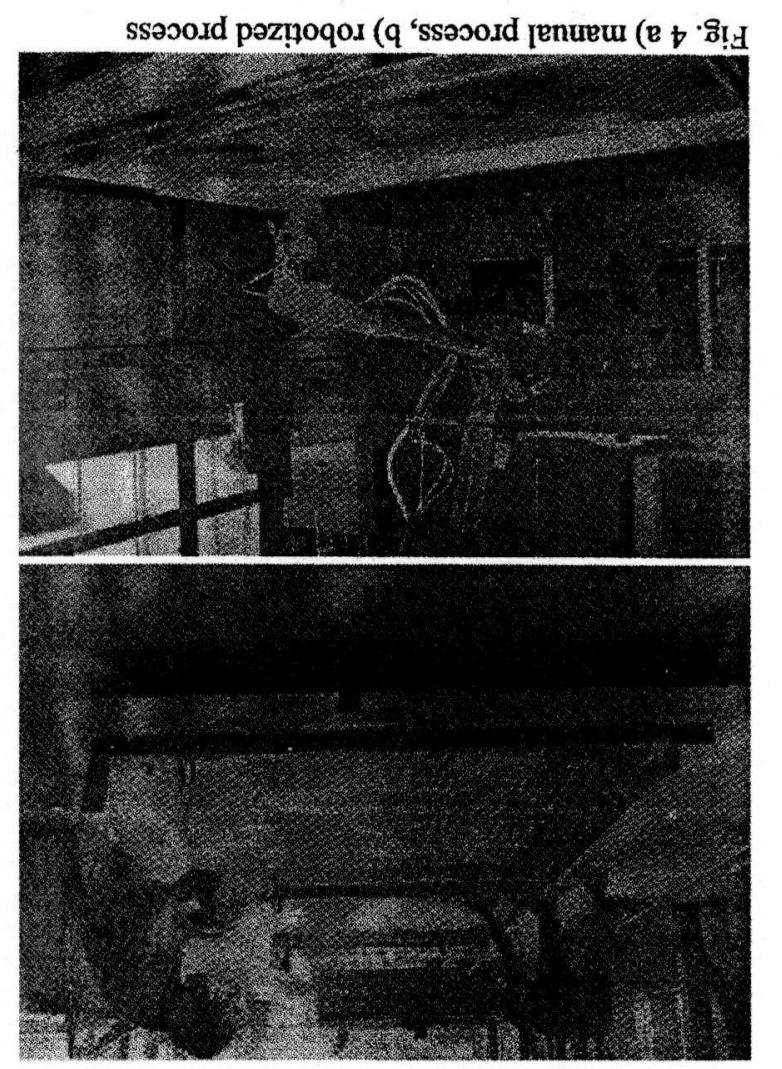

\section{SYSTEM ARCHITECTURE}

The manufacturing of a GRC panel goes through several stages: 1) mold preparation: the placement of clamps for later assembly on site, 2) "spraying/compacting, 3) hardening, 4) panel extraction from the mold, and 5) curing. The mold is manually made in wood, however with the appearance of new materials automation of this stage could also be considered [4]. As spraying/compacting is the most critical one automation has focused on it.

As mentioned above, the objective was the automation of both the spraying and compacting processes, 
but after first experiments with the spraying cell it followed that the quality of the spraying was so good that intermediate compacting stages could be eliminated.

Fig. 5 shows a scheme of the cell. A brief description of the equipment involved in the whole manufacturing process is now presented.

- Spraying robot: 6 DOF commercial robot. It is placed upside down on the center of the cell. It is capable of being controlled in real-time from an external computer.

- Spraying gun: concentric spraying gun attached to the tip of the robot with a power of up to $28 \mathrm{~kg} / \mathrm{min}$.

- An electronic pump controls mortar parameters.

- On-line main computer: industrial PC connected to 1) the robot Computer Link, 2) the field-bus PLCs network, and 3) the off-line computer. It performs a monitoring of the equipment status and the scheduling of parts to be manufactured on a working day.

- Off-line computer: PC with a commercial CAD package (AUTOCAD v12). This is the PC where the $\mathrm{CAD}$ process is performed in the design office.

- Programmable Logic Computers (PLCs): three PLCs, connected to the on-line main computer via network, control respectively the electronic pump, the hopper \& mixer and the roller conveyors.

- 3 meters wide roller conveyors are used to move the molds in the factory.

Fig. 6 presents the control system structure of the developed GRC manufacturing system. The more important aspect that characterizes the system is the integration of CAD with CAM to cope with small batch size production of different panels. Molds are designed on a commercial CAD environment with access to information of the manufacturing tools and design rules of the product. The information generated by the CAD environment is concerning 3D drawings and product features. This raw information is processed through an off-line module, similar to computed distributed system used in

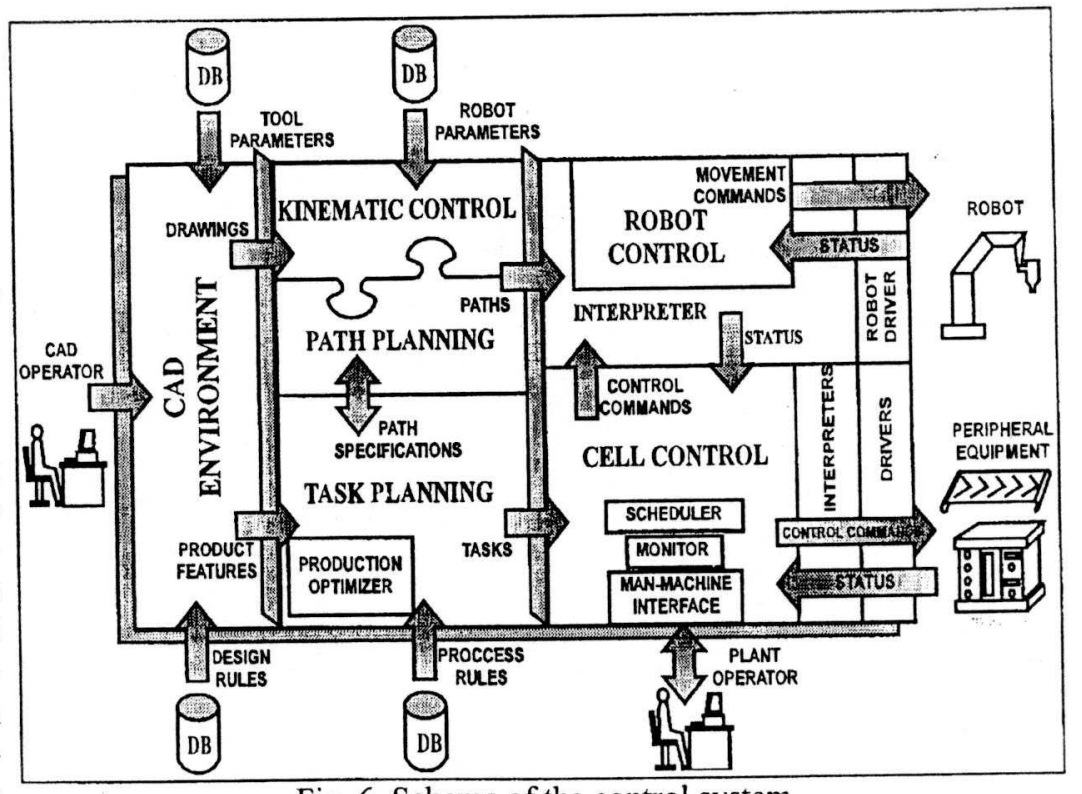

Fig. 6 Scheme of the control system
Fig. 5 Scheme of the cell

[5]. Three interrelated sub-modules form the module: robots kinematics control, path planning and task planning. Each of the sub-module generates commands for the on-line equipment in the manufacturing cell: robot, computers, PLCs, etc.

A commercial 6 DOF robot was selected as the spraying machine. A manual programming of the robot was impossible due to the complexity and the great number of different panels. Therefore off-line programming was adopted. In this sense, real-time communication with the robot has been one of the key factors during robot selection.

\section{CAD ENVIRONMENT}

One of the advantages of the system is the integration of CAD with CAM, and specially the automatic robot path-planning directly from $3 \mathrm{D}$ CAD drawings. First of all the CAD operator makes a detailed 
drawing of the desired building facade. In order to facilitate the design, a series of software utilities are included in the menu bar of AUTOCAD. Once the facade has been drawn under solid modeling, the automatic facade partitioning into elementary panels is performed (Fig. 7). For this purpose it is necessary to consider process specifications, i.e. maximum size of panels to be manufactured, windows and doors sectioning, etc. Finally from the elementary panels their molds are generated.

For each panel, the operator must specify various general process and tool parameters that normally remain fixed for several panels. These parameters include: spraying cone angle, rated spraying flow, number and type of layers (bottom or side, thickness, material), type and position on insulators and clamps, etc. Finally, the operator can launch the automatic generation of layers in the CAD environment and then the robot path planning procedure.

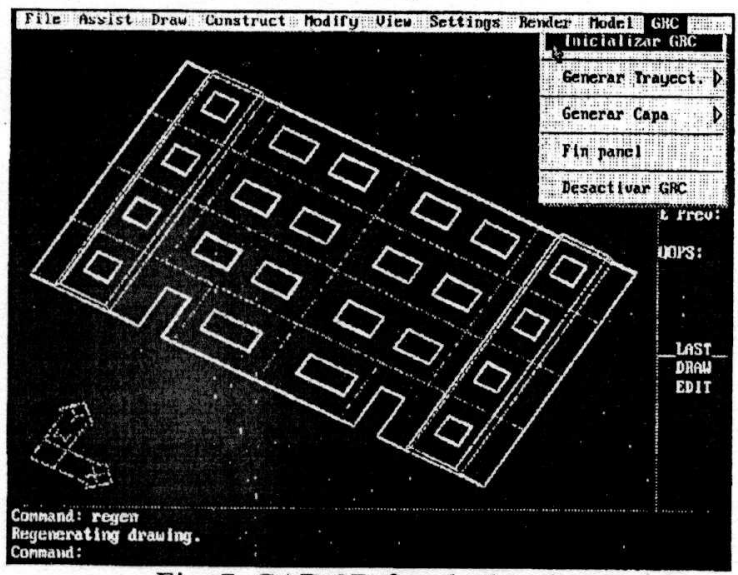

Fig. 7 CAD 3D facade drawing

\section{SPRAYING RULES}

The path planning process depends mainly on some spraying rules that were obtained from a careful study of manual spraying, with an empirical parametrization of the spraying cone [6] They are:

- Spraying must be performed perpendicular to the surface whenever possible.

- The surface to be sprayed is divided in parallel spraying paths. Path width is adjusted modifying the spraying distance to obtain an integer number.

- Slopes in the bottom of the mold less that $7 \mathrm{~cm}$ are ignored in the generation of the spraying orientation.

- Bottom and sides of the mold are sprayed in different stages.

- In order to reinforce the panels corners and edges the amount of material sprayed in these zones must be greater than in other ones.

- The spraying of the bottom is done alternatively in perpendicular directions for consecutive layers

- Spraying direction of the gun in the bottom is interpolated if the angle between bottom planes is more than $60^{\circ}$.

\section{ROBOT PATH PLANNING ROBOT PATH GENERATION}

There are several steps in the automatic robot path planning algorithm. This algorithm receives data of the mold 3D drawing together with the spraying parameters, and it generates the real robot path and spraying gun commands (Fig. 9). The path planning algorithm works in first place with a spraying gun path only and then transforms it to a robot path [7]. From the mold data (Fig. 9a) a theoretical spraying gun path is calculated (Fig. 9b).

In order to ensure the homogeneity of the layer the paths are parallel straight lines segments on plane surfaces. Their two extreme points would be enough to specify the position in the mold where the center of the spraying cone base has to be placed, but more information is needed to determine the orientation and the distance from the mold to the gun's tip. Hence, for each point on the surface of the layer it is necessary to calculate another point indicating where the gun's tip is to be located. A vector called robotto-panel vector vr-p (Fig. 9b) expresses the distance and orientation of the gun.

Once the straight segments needed to spray a whole panel have been generated, the objective is to obtain a real spraying gun path (Fig. 9c). The path starts is $\mathrm{S}$ and ends in $\mathrm{G}$ (both are automatically selected) and includes intermediate points (II and I2) where the spraying has to be stopped and restarted. This step consists in calculating the optimum way to track the straight segments, considering the restrictions in order to establish the best solution: minimum number of gun stops, minimum robot kinematics configuration changes and vertical progress of the path: the spraying must be done upwards in the slopes. The resulting trajectory has a wavy pattern in order to obtain better uniformity. The theoretical study done in [8] supports our experimental results of minimal variation of the accumulated film thickness on the mold surface.

To obtain the theoretical robot path (Fig. 9d) a kinematics study of the generated real spraying gun path is performed. The straight segments are subdivided in equally spaced (about $10 \mathrm{~cm}$ ) spraying points for the robot. Moreover, to avoid singular robot positions several modifications of these points are made, e.g. changing the orientation of the gun in conflict areas and axes [9].

\section{KINEMATICS ROBOT PATH}

The objective is to position the robot with the appropriate orientation over the panel (Fig. 9e). This is the step where for the first time the robot kinematics is analyzed. Because of the manufacturing process three additional restrictions, one static and two dynamic, have to be considered: the path must be continuous in orientation to avoid sudden changes in the 


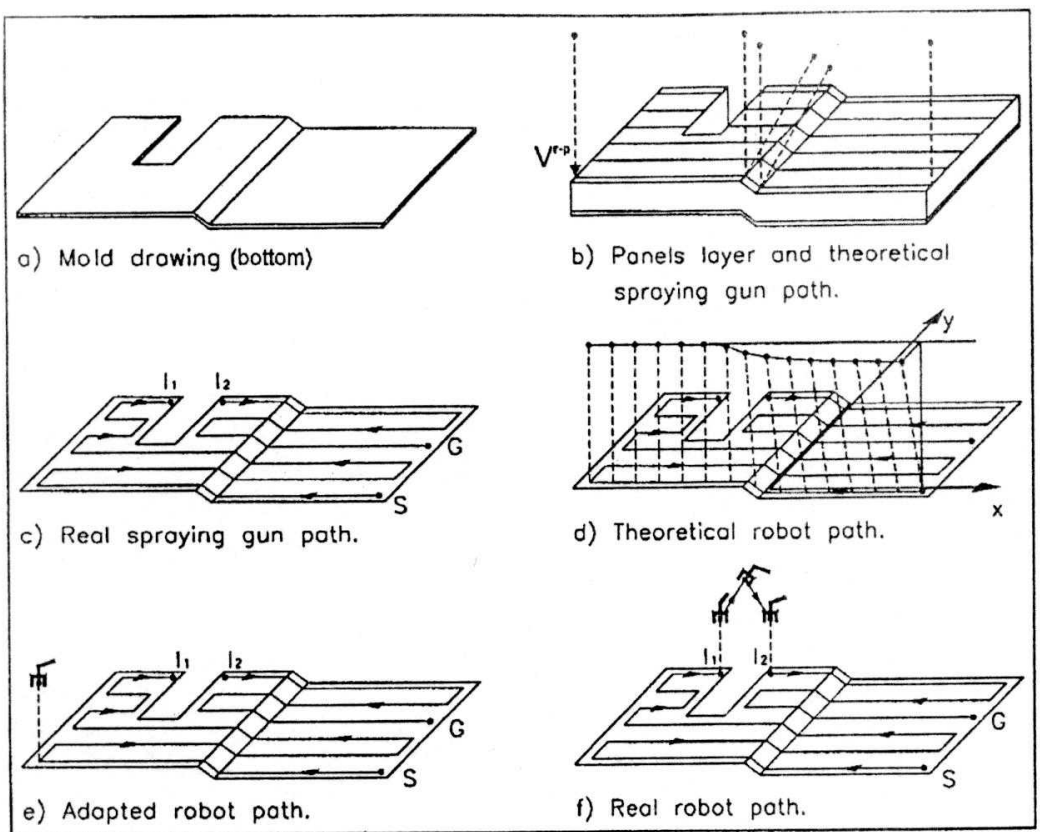

Fig. 8 Robot path planning steps

time consuming computational work has been done before in the path planning stage, the robot control module is fairly simple. It reads the path files and sends the position commands to the robot controller through a dedicated serial link. The status of the command execution is received and taken in consideration. In special situations in which an unexpected path has to be generated a simplified version of the connection control algorithm is used.

The monitor displays on the manmachine interface all the common events as well as any other unexpected event that may influence the manufacturing performance. Messages about plant status are sent directly from the sensors to orientation of the spraying gun with negative influence in the path quality, and due to the extreme fragility of the glass fiber that goes to the spraying gun along the arm of the robot. In general the robot must move following straight line segments in Cartesian coordinates. This implies the existence of multiple singular points that have to be avoided.

To generate the kinematics robot path several sequential steps are performed. First, to fulfill continuous orientation path restrictions an algorithm to smooth the degree of change of the orientation is executed. The next step is the fulfillment of the glass fiber orientation restriction in Cartesian coordinate movements.

The final step in the path planning is the generation of a real robot path (Fig. 9f) through the use of robot approach and retreat algorithms. This algorithm moves the robot between two distant points, avoiding collision with the mold. The real robot path is the final result of the path planning and is saved in path files that are later used as source information for the on-line control. Simultaneously with the path more specific information is also generated: task secuences, number and type of layers, positions where to stop/start gun, PLCs commands to be executed by the peripheral equipment, etc.

\section{ON-LINE CONTROL OF CELL}

The on-line control module executes the path files that have been generated in the preceding stages. Four different processes run sequentially on the online computer: the scheduler, the robot control, the monitor of events and the man-machine interface.

The scheduler works as the core engine [17]. Reading task files and event information from the monitor, it decides which action to perform. Since all the

the PLCs, and from these to the network board events registers. Here the monitor withdraws them. Fig. 9 shows the man-machine interface during online control of the cell. Different user friendly menus allow the interaction with the cell: start, pause, resume or halt production. These commands are received by the monitor, which passes them to the scheduler to begin proper actions. The status of different equipment of the cell (molds, mixer, robot, conveyors, etc.) is displayed through color code. A message bar on the bottom part of the screen shows any useful information about the processes evolution.

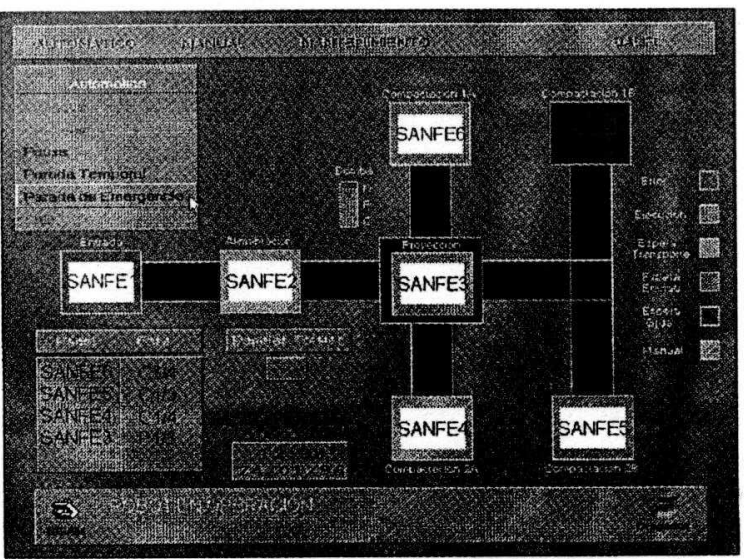

Fig.9 Man-machine interface

\section{EVALUATION AND COMPARATIVE STUDY}

To evaluate the achieved improvements the developed robotized system is compared to traditional manual manufacturing. The comparative study is based on two key factors: product quality and overall productivity [10].

The main criteria for product quality evaluation are layers uniformity and structural parameters. The 
layers sprayed by the robot are more uniform than the layers obtained by manual spraying, mainly because the robot describes straight line paths in a specific direction over the mold surface. The manual sprayed panel is more irregular mainly because the reach of the worker is not large enough to encompass all the panel area, which has an average of $5 \times 3 \mathrm{~m}$. The robot also sprays with more uniformity due to greater pressure of the spraying gun. The pressure is more than the double of the manual one, accomplishing a better mixing and compacting of the glass fiber with the mortar. This fact is very important, because it allows eliminating all intermediate compacting between layers, saving time and labor.

Mechanical structural features are also important for the quality evaluation of GRC panel manufacturing. The uniformity of spraying by robot can also be observed on flex-traction tests results. The strength of the test piece measured in longitudinal and transversal directions is very similar for robotized and manual manufacturing.

The panel manufacturing time cycle can be divided into two different phases: a) mold design and drawing followed by path planning, and b) manufacturing in factory. The first phase, which is performed completely off-line, can be done in the technical office. If there are several panels with similar geometry they can be grouped together and can be generated quicker with slight modifications to the first one, taking an average of $20 \mathrm{~min}$. per panel.

On the other hand, the robot spraying times are inferior to the manual ones because the robot is spraying with a $28 \mathrm{~kg} / \mathrm{min}$ gun versus a $12 \mathrm{~kg} / \mathrm{min}$ manual gun.

The huge advantage of robotic spraying is the elimination of intermediate compacting between layers, as the mortar is sprayed with higher pressure and uniformity. This also eliminates transportation times together with robot idle stages, increasing significantly the whole productivity for each panel.

\section{CONCLUSIONS}

The developed system (Fig. 10) presents a new step towards fully automatic prefabricated manufacturing. The development of this system has shown some of the great advantages that automation can bring into quality and factory productivity in an off-site manufacturing process of construction industry: a) improvement in layers and thickness uniformity, b) similar mechanical strength test results, c) elimination of all intermediate compacting between layers, d) productivity increase, and e) materials saving.

This research project proves that new robotic technologies can be introduced in construction industry with good results. The research done has also contributed to a better understanding of the production process and to search for new ways of automation.

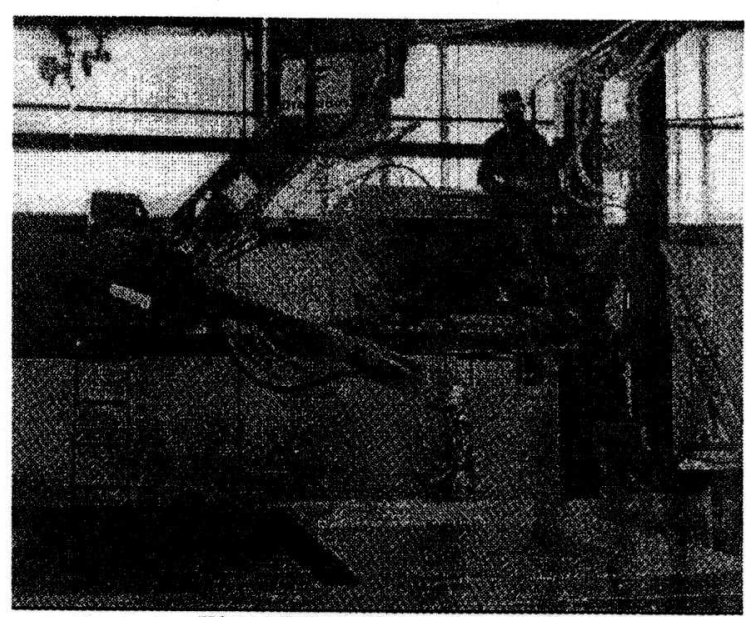

Fig. 10 Developed system

\section{REFERENCES}

[1] Balaguer, C., Pastor, J.M. "Robotized system of GRC panels for construction industry", 10th Int. Symposium on Automation and Robotics in Construction (ISARC'93), Houston (USA), 1993.

[2] Rembold, U., Nhaji, B.O. and Storr, A., "Computer Integrated Manufacturing and Engineering", Addison-Wesley, 1993.

[3] Yamazaki, Y., "Conceptual design and construction planning system for Computer Integrated Construction", Automation in Construction, Vol. 1, No. 1, 1992.

[4] Viswanathan, A., Jouaneh, M., Datseris, P. and Palm, W., "A manufacturing system for automated production of polystyrene molds", IEEE Robotics \& Automation Magazine, Vol. 3, No. 3, 1996.

[5] Cheng, H. H. "Stacking irregular-sized packages by a robot manipulator", IEEE Robotics \& Automation Magazine, Vol. 2, No. 4, 1995.

[6] Goodman, E. D. and Hoppensteadt, L.T.W., "A method for accurate simulation of robotic spray application using empirical parametrization", IEEE International Conference on Robotics and Automation, (ICRA'91), Sacramento (USA), 1991.

[7] Balaguer, C., Pastor, J.M., Peñin, L.F. and Rodriguez, F.J., "Automatic robot path planning for GRC spraying cell", 11th International Symposium on Automation and Robotics in Construction (ISARC'94), Brighton (UK), 1994.

[8] Antonio, J. K., "Optimal trajectory planning for spray coating", IEEE International Conference on Robotics and Automation, (ICRA'94), 1994.

[9] Everett, L.J., Colson, J.C. and Mooring, B.W., "Automatic singularity avoidance using joint variations in robot task modification", IEEE Robotics \& Automation Magazine, Vol. 1, No. 3, 1994.

[10]Balaguer, C., García, A., Pastor, J.M. and Peñin, L.F., "Evaluation and comparative study of robotics vs manual spraying of GRC panels", 12th International Symposium on Automation and Robotics in Construction (ISARC'95), Warsaw (Poland), 1995. 